DOI:10.24193/tras.56E.3

Published First Online: 02/28/2019
Abstract

The paper presents a direct international comparison of the existing fiscal decentralization systems of European Union member states (hereinafter EU-28), which are also signatories of the European Charter of Local Self-Government (hereinafter ECLSG or Charter). The results of the benchmarking show that the conceptual index of fiscal decentralization (hereinafter (IFD) is an appropriate tool for direct international comparison of different fiscal decentralization systems, while allowing measurements of their effectiveness, as there is a direct link between the CIFD and the basic principles of the ECLSG.

The CIFD is an upgrade of classical methods (indicators) used to measure fiscal decentralization, and, at the same time, reflects the consideration of the selected basic principles of the ECLSG. The CIFD consists of five indicators, four of which represent quantified selected basic principles of the ECLSG, and the fifth indicator represents the number of lower management levels. Thus, with the use of CIFD, the EU-28 obtained definite levels of fiscal decentralization, while, due to the established direct link between the CIFD and the ECLSG guidelines, the indices in the index structure themselves indicated either greater or lesser compliance of their fiscal decentralization systems with the selected basic principles of the Charter.

The paper concludes with a simulation of an empirical conceptual model of fiscal decentralization (hereinafter CMFD) which is now offering the opportunity to identify critical points or shortcomings of the existing fiscal decentralization system in each country, limiting or even eliminating them with the aim of achieving a highly efficient system of fiscal decentralization.

Keywords: fiscal decentralization, European Charter of Local Self-Government, international comparison, fiscal decentralization index, model of fiscal decentralization.

\section{DIRECT INTERNATIONAL COMPARISON OF EU MEMBER STATES FISCAL DECENTRALIZATION SYSTEMS WITH THE CONCEPTUAL INDEX OF FISCAL DECENTRALIZATION (CIFD) IN THE CONTEXT OF EUROPEAN CHARTER OF LOCAL SELF-GOVERNMENT (ECLSG)}

\section{Mateja FINŽGAR}

PhD Student, Faculty of Economics and Business, University of Maribor, Maribor, Slovenia

E-mail: matejafinzgar@gmail.com

Boštjan BREZOVNIK (corresponding author)

Associate Professor, PhD, Faculty of Law, University of Maribor, Maribor, Slovenia

E-mail: bostjan.brezovnik@um.si 


\section{Introduction}

The literature in the field of fiscal decentralization evinces no consensus on how to unify the mutually different fiscal decentralization systems of individual countries. Having reviewed the relevant literature related to fiscal decentralization, we find that authors primarily present their findings to the public from a theoretical perspective and do not use any suitable research reflecting the real situation regarding fiscal decentralization in individual countries, and, at the same time, enable their direct comparison. Namely, such research is based on the use of, more or less, classical and auxiliary indicators, which only indirectly describe the relationships between individual government strata. Moreover, such indicators are not appropriately embedded in the context of the ECLSG's most important guidelines, the fundamental multilateral legal instrument in the field of local self-government in Europe.

The ECLSG was adopted by the Council of Europe in 1985 but did not come into force until 1988, when it was ratified by the parliaments of its initial four member states, later joined by other member states. By signing the Charter, the member states of the Council of Europe unified awareness in the belief that one of the ways to achieve unity is by making agreement at the local community level; simultaneously, they undertook to implement the guidelines, or so-called fundamental principles, set forth in the Charter in their legislative regulation of local self-government.

In this paper, we focus on the selected fundamental principles in the Article 9 of the ECLSG that relates to local community financial resources, which we believe to represent the foundation for establishing an effective fiscal decentralization ${ }^{1}$ system in every member state of the Council of Europe; these systems should be rooted in the principles of flexibility, financial resources being commensurate with responsibility, autonomy and equality.

The ECLSG steers European countries in the common direction of establishing effective fiscal decentralization systems based on determined guidelines for the development of local communities. To discover which of the analyzed countries have formed more and which have formed less effective fiscal decentralization systems, we must make a direct international comparison of their established fiscal decentralization systems. We believe that better compliance with the fundamental principles of the ECLSG results in more effective fiscal decentralization systems in individual countries. Our direct international comparison of existing EU-28 fiscal decentralization systems was made using the CIFD, which is comprised of the following five indicators:

- $\mathrm{IF}_{\mathrm{i}}$ : the flexibility indicator for country $\mathrm{i}$ with values between 0 and 1 ;

1 The benefits and importance of fiscal decentralization have been discussed at length in the recent literature by Bole and Jere (2005), Brezovnik and Oplotnik (2003), Cheikbossian (2008), Sandler and Tschihart (1997), Slavinskaite (2015) and Vo (2015); thus, we will not insist on these issues any further. 
- IS: the indicator for local community financial resources being commensurate with local community responsibility in country i with values between 0 and 1 ;

- $\mathrm{IA}_{\mathrm{i}}$ : the autonomy index for country $\mathrm{i}$ with values between 0 and 1 ;

- II: the equalization index for country $i$ with values between 0 and 1 ; and

- $\mathrm{IR}_{\mathrm{i}}$ : the indicator for the number of local communities in country $i$ with values between 0 and 1 .

In this way, we obtained a result for the analyzed countries (European Union member states) in the form of determinable levels of fiscal decentralization, whereby CIFD's indicators alone point to higher or lower levels of fiscal decentralization system compliance with the selected fundamental principles of the ECLSG. Here, we assume that CIFD represents a model of the real situation and that it is comprised of such parameters/indicators whose value can change in different observation periods. Such indicators were used to prepare a simulation of an empirically based CMFD, which reflects high compliance with the fundamental principles of the ECLSG and can be used by member states (that is, signatories to the Charter) as a basis for improving the effectiveness of their existing fiscal decentralization systems.

\section{Overview of theoretical baselines and comparisons of fiscal decentralization systems}

The establishment of a decentralized public sector should provide a solution for the conceptual public problem of choice, whose central issue is connected to the ability to effectively allocate responsibilities, powers and financial resources from the national to the local level, keeping in mind local population heterogeneity throughout the world (Finžgar and Oplotnik, 2013, p. 655). It is assumed that local authorities in individual countries will only be effective once given suitable powers and access to financial resources formed of a suitable combination of their own financial revenue and intergovernmental transfers (Boex, 2009, p. 8). The traditional, first generation of literature related to the concept of fiscal decentralization is provided by pioneers in this area, such as Tiebout (1956), Musgrave (1959), Buchanan (1965), Olson (1969), Samuelson (1954) and Oates (1972).

First-generation authors present their conclusions, suggestions and findings regarding fiscal decentralization from a theoretical perspective without suitable quantitative or qualitative empirical research reflecting the actual situation regarding fiscal decentralization in individual countries and, consequently, enable their direct comparison. In spite of the gap between the authors' theoretical findings regarding assumptions concerning the effectiveness of local authority/decentralized unit work and practice in many developed countries, they have been used as the central foundation for further research in the field of fiscal decentralization.

The awareness that fiscal decentralization systems must be looked at from a broader perspective has strongly increased amongst new-age authors or representatives of the second generation in the field of fiscal decentralization, such as Weingast (1995), 
Seabright (1996), Basley and Coate (2003), and Wagner (2007). The second-generation attributes an important role to citizens who, by cooperating in public matters, impact decision making in relation to the environment they live in and, consequently, enable more effective local authority operation in relation to the supply of public goods and the implementation of general administration responsibility.

Many published quantitative and qualitative studies carried out by second-generation and other new-age authors are based on the use of classical methods/indicators, which only indirectly describe relationships in individual local authority/decentralized units and/or relationships between the central government and local authority/ decentralized unit level. As such, the results of studies obtained on this basis are insufficient in terms of unambiguously presenting the actual situation in terms of fiscal decentralization in individual countries, making direct comparisons difficult. As aforementioned, the literature published thus far does not provide a common consensus regarding how to unify the different fiscal decentralization systems of individual countries.

\section{Direct international comparison of fiscal decentralization systems utilizing the conceptual index of fiscal decentralization (CIFD)}

Each EU-28 member state has developed its own model and operational structure for its national government and local authorities/decentralized units. Fiscal decentralization is, therefore, in practice and theory, treated differently in each country, meaning that the government system differs from one country to another. A direct comparison of such very different government systems providing a realistic presentation of fiscal decentralization in individual countries in a selected time period represents a major challenge. By forming the CIFD we wanted to unify the different fiscal decentralization systems. By finding a common denominator, that is, CIFD, for such systems, it can be verified which systems were created to achieve higher and which were created to achieve lower levels of effectiveness. The establishment of real effectiveness of individual fiscal decentralization system can assist in outlining a country's strengths and weaknesses. Being aware of its strengths and weaknesses, individual countries can appropriately upgrade their existing fiscal decentralization system in a direction which achieves increased effectiveness.

CIFD includes a total of five indicators, four of which directly reflect the fundamental principles of the ECLSG, and relate to the local authority/decentralized unit financial resources. We believe that the observation of the selected fundamental principles of the ECLSG is a basis for the establishment of more effective fiscal decentralization systems. European countries also differ significantly in terms of the number of local authorities/decentralized units, and this is a consequence of their different primarily historical, as well as geographical and cultural, backgrounds. The number of local authorities/decentralized units thus presents CIFD's fifth selected indicator. Initially, CIFD is presented as a figure, followed by a formula, including presentation of every indicator. 


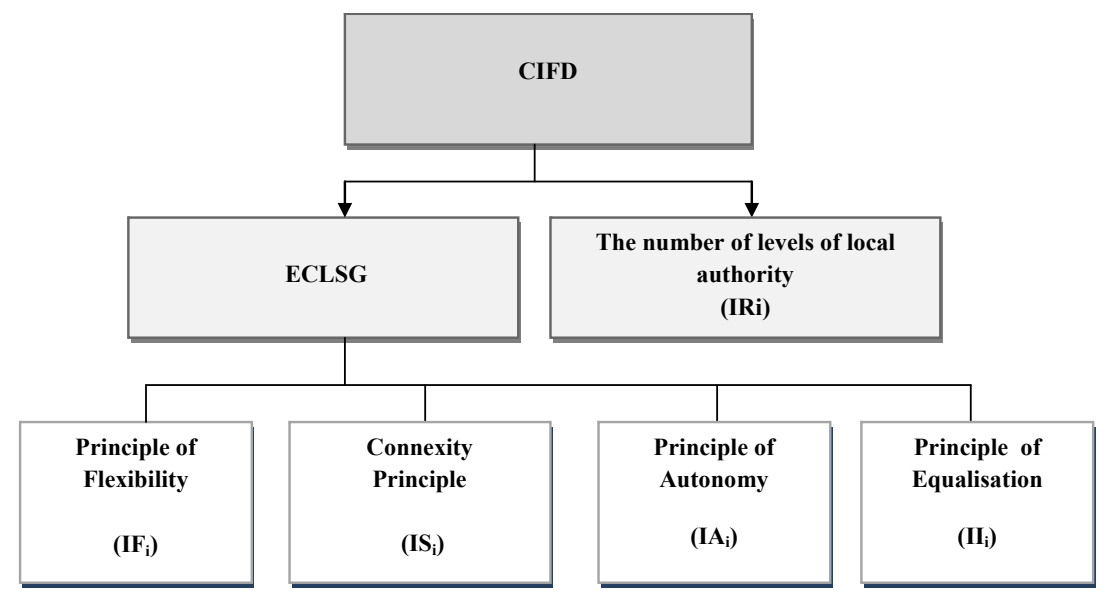

Figure 1: Structure of the CIFD

Source: The authors

As seen in Figure 1, CIFD is comprised of two parts: the first part is presented in terms of the quantified fundamental principles of the ECLSG (indicators $\mathrm{IF}_{\mathrm{i}^{\prime}} \mathrm{IS}_{\mathrm{i}^{\prime}} \mathrm{IA}_{\mathrm{i}^{\prime}}$ $\mathrm{II}_{\mathrm{i}}$ ), while the other presents the number of levels of local authority/decentralized units (indicator $\left.\mathrm{IR}_{\mathrm{i}}\right)^{2}$. The CIFD's formula is as follows:

$$
\mathbf{C I F D i}=\mathbf{W 1} \times \mathbf{I F i}+\mathbf{W 2} \times \mathbf{I S i}+\mathbf{W 3} \times \mathbf{I A} \mathbf{i}+\mathbf{W 4} \times \mathbf{I I} \mathbf{i}+\mathbf{W 5} \times ， \quad(1), \text { where: }
$$

CIFDi - the conceptual index of fiscal decentralization of country $\mathrm{i}$;

$\mathrm{W}_{1-5}$ - importance weighting of individual of CIFD indicators, whereby total value $\mathrm{W}=1$;

$\mathrm{IF}_{\mathrm{i}} \quad$ - flexibility indicator of country $\mathrm{i}$ with values between 0 and 1 ;

$\mathrm{IS}_{\mathrm{i}} \quad$ - the indicator of local government financial resources being commensurate with country i's responsibilities with values between 0 and 1 ;

$\mathrm{IA}_{\mathrm{i}} \quad$ - the indicator of autonomy for country $\mathrm{i}$ with values between 0 and 1 ;

$\mathrm{II}_{\mathrm{i}} \quad$ - the indicator of equality for country $\mathrm{i}$ with values between 0 and 1 ;

$\mathrm{IR}_{\mathrm{i}} \quad$ - the indicator of the number of local government bodies in country $\mathrm{i}$ with values between 0 and 1 .

During the preparation of our study, we did not find authors who have, thus far, made an indirect comparison of the fiscal decentralization systems of our selected countries using the Fiscal Decentralization Index (hereinafter FDI), a system which ranks indicators according to importance weighting, represented as an individual FDI indicator. With CIFD, we made a step forward and ranked CIFD's formula indicators according to importance weighting (W1-5) whereby the total value of impor-

2 The selected time period for the calculation of individual indicators of the CFDI is between 2013 and 2015 and it depends on the selected segment and country and the data bases that were or are available for individual segments and countries. 
tance weighting for all indicators equals $1(\mathrm{~W}=1)$. The latter is shown in Figure 2 . The result obtained with CIFD represents a determinable fiscal decentralization level for the analyzed countries and thereby a tool for the implementation of a direct international comparison of its already existing fiscal decentralization systems.

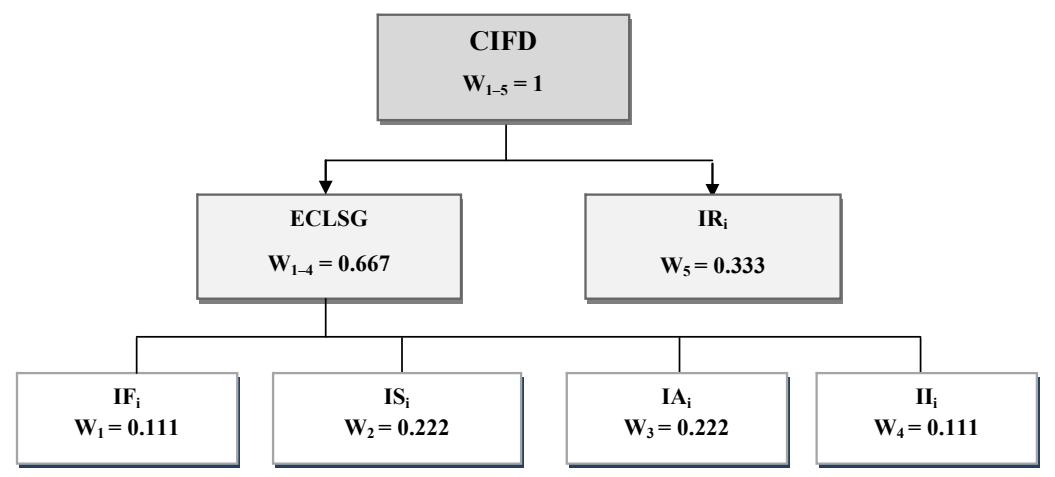

Figure 2: Ranking of CIFD indicators according to importance weighting

Source: The authors

CIFD's individual indicator weighting is based on knowledge obtained to date in the field of fiscal decentralization. As already emphasized, the ECLSG is the most important multilateral legal document in the field of local self-government in Europe. In terms of local authority/decentralized unit legal regulation, member states, the signatories of the ECLSG, should consider its fundamental principles. On this basis, we assume that the importance weighting of the selected fundamental principles of the ECLSG in CIFD amount to more than half, that is, 0.667 or $2 / 3$. We believe that observing and/or abiding by the fundamental principles of the ECLSG is vital for an individual country in order to achieve the most suitable level of decentralization in its environments and, at the same time, represents the basis for the establishment of more effective fiscal decentralization systems in light of improved transparency, comprehensiveness and simplicity.

CIFD's remaining importance weighting in the amount of 0.333 or $1 / 3$ represents the number of local government bodies. In comparison with the fundamental principles of the ECLSG, the importance weighting for this indicator is smaller, as it also arises from the provisions of the ECLSG. The third paragraph of Article 4 of the ECLSG states that public responsibilities shall generally be exercised, in preference, by those authorities which are closest to the citizen. Since the number of local authority bodies affects the fiscal decentralization system policy implementation in individual countries, the importance weighting of such CIFD indicators must, on the one hand, not be negligible, and on the other hand, not exceed the importance weighting assigned to the fundamental principles of the ECLSG. This Charter is the basis for the formation of local authorities/decentralized units in individual countries. 
Furthermore, CIFD determines the importance weighting for each of the selected fundamental principles included in the ECLSG. The total sum of importance weightings of all four indicators representing the quantified foundation of the ECLSG does not exceed CIFD's importance weighting of the ECLSG (0.667 or 2/3). We assume that two of the fundamental principles of the ECLSG, that is, the 'principle of local government financial resources being commensurate with responsibilities' and 'the principle of local authority autonomy' represent greater importance weighting when forming effective fiscal decentralization systems in individual countries than the other two fundamental principles, 'the principle of flexibility' and 'the principle of equality'. CIFD's importance weights IS $_{i}$ and IA $_{\mathrm{i}}$ represent 0.222 each, whilst the importance weight for the other two indicators, $\mathrm{IF}_{\mathrm{i}}$ and $\mathrm{II}_{\mathrm{i}^{\prime}}$ is evidenced at 0.111 each.

With our 'principle of local government financial resources being commensurate with responsibilities' $\left(\mathrm{IS}_{\mathrm{i}}\right.$ ) we establish whether individual countries are able to cover local authority expenditure with centrally-earmarked financial resources. In the observation period, we believe coverage of total local authority expenditure on carrying out tasks under their responsibility with disclosed revenue is the foundation for the formation of effective and sustainable fiscal decentralization systems in each individual country. The same CIFD importance weighting is also attributed to the 'principle of local authority autonomy'.

The 'principle of local authority autonomy' states that local authorities are entitled to adequate financial resources of their own, which they may freely dispose of within the framework of their powers, and autonomously attribute taxes and charges (Article 9 of the ECLSG). An autonomous financial resource within each country for individual local authorities is their ability to determine tax rates and relief without national government consultation (EC 2012 and 2016). IA $_{\mathrm{i}}$ was used to establish the share of autonomous, local authority financial resources disposed of within individual countries. We believe that the issue of autonomy, considered one of fiscal decentralization analysis' key issues, is also an important factor for establishing effective fiscal decentralization systems in individual countries.

On the other hand, smaller CIFD importance weightings are attributed to the 'principle of flexibility' $\left(\mathrm{IF}_{\mathrm{i}}\right)$ and the 'principle of equality' $\left(\mathrm{II}_{\mathrm{i}}\right)$. When calculating individual CIFD indicators, values presented in detail in a continuation of this paper's work, we found that even if a country shows one of the highest $\mathrm{IF}_{\mathrm{i}}$ values, indicating a high number and variety of financial resources available to local authorities in an individual time period, it does not mean that it manages to fully cover all of its stated expenses with its total revenue (e.g., Belgium). Likewise, we found that even if a country shows one of the highest $\mathrm{II}_{\mathrm{i}}$ values, with national government transfers representing an important share of their total revenue, it still does not mean that its local authorities earned more revenue than expenses faced in the observation period (e.g., Malta, the Netherlands, United Kingdom, Lithuania, Belgium, Poland, Denmark, and Estonia). As a result, local government in these countries must incur debts for the purpose of implementing tasks under their responsibility and accumulate deficits, which can affect their autonomy in the long run. 
It should also be noted that the systems of fiscal decentralization of the analyzed countries will be presented by using innovative tables in the selected period of time, which will be moving between 2013 and 2015 and will depend on the selected segment and country depending on the data bases that were available for individual segments and countries. The obtained levels of fiscal decentralization for EU-28 countries by means of CIFD are presented in Table 1.

Based on the calculated fiscal decentralization levels of the analyzed countries by means of CIFD, we found that the highest fiscal decentralization levels of all EU-28 countries are shown by Belgium (0.75), Italy (0.72), France (0.71), United Kingdom (0.71) and Poland (0.71). On the other hand, the lowest fiscal decentralization levels are present in Latvia (0.41), Cyprus (0.42), Estonia (0.42), Malta (0.45) and Slovenia (0.47). We assume that the closer the value of CIFD for an individual country is to 1 , the higher the level of fiscal decentralization achieved by the analyzed fiscal decentralization system will be. The borderline value between countries with high levels of fiscal decentralization (Fiscal Decentralization Group 1; hereinafter: FDG1) and countries with a medium level fiscal decentralization (Fiscal Decentralization Group 2; hereinafter: FDG2) is represented by 0.67 or $1 / 3$ of all countries observed. The countries whose CIFD value is equal to or lower than the calculated average of CIFD value for all EU-28 countries (0.59) are ranked in the group of countries with low fiscal decentralization levels (Fiscal Decentralization Group 3; hereinafter: FDG3).

Table 1: Calculation of fiscal decentralization levels for EU-28 countries by means of CIFD

\begin{tabular}{|c|c|c|c|c|c|c|c|c|c|c|c|}
\hline States & W1 & IFi & W2 & $\mathrm{ISi}$ & W3 & IAi & W4 & IIi & W5 & IRi & CIFD \\
\hline BEL & 0.11 & 0.91 & 0.22 & 0.99 & 0.22 & 0.10 & 0.11 & 0.63 & 0.33 & 1.00 & 0.75 \\
\hline ITA & 0.11 & 0.91 & 0.22 & 1.00 & 0.22 & 0.07 & 0.11 & 0.45 & 0.33 & 1.00 & 0.72 \\
\hline FRA & 0.11 & 0.82 & 0.22 & 0.96 & 0.22 & 0.17 & 0.11 & 0.34 & 0.33 & 1.00 & 0.71 \\
\hline UK & 0.11 & 0.73 & 0.22 & 0.99 & 0.22 & 0.00 & 0.11 & 0.69 & 0.33 & 1.00 & 0.71 \\
\hline POL & 0.11 & 0.82 & 0.22 & 0.99 & 0.22 & 0.00 & 0.11 & 0.57 & 0.33 & 1.00 & 0.71 \\
\hline GER & 0.11 & 0.91 & 0.22 & 1.00 & 0.22 & 0.04 & 0.11 & 0.27 & 0.33 & 1.00 & 0.69 \\
\hline SPA & 0.11 & 0.82 & 0.22 & 0.95 & 0.22 & 0.00 & 0.11 & 0.35 & 0.33 & 1.00 & 0.67 \\
\hline FIN & 0.11 & 0.73 & 0.22 & 0.96 & 0.22 & 0.41 & 0.11 & 0.30 & 0.33 & 0.70 & 0.65 \\
\hline NET & 0.11 & 0.82 & 0.22 & 0.98 & 0.22 & 0.00 & 0.11 & 0.70 & 0.33 & 0.70 & 0.62 \\
\hline ROM & 0.11 & 0.64 & 0.22 & 1.00 & 0.22 & 0.00 & 0.11 & 0.84 & 0.33 & 0.70 & 0.62 \\
\hline HUN & 0.11 & 0.73 & 0.22 & 1.00 & 0.22 & 0.00 & 0.11 & 0.68 & 0.33 & 0.70 & 0.61 \\
\hline DEN & 0.11 & 0.82 & 0.22 & 1.00 & 0.22 & 0.01 & 0.11 & 0.57 & 0.33 & 0.70 & 0.61 \\
\hline GRE & 0.11 & 0.73 & 0.22 & 1.00 & 0.22 & 0.00 & 0.11 & 0.68 & 0.33 & 0.70 & 0.61 \\
\hline POR & 0.11 & 0.91 & 0.22 & 1.00 & 0.22 & 0.00 & 0.11 & 0.42 & 0.33 & 0.70 & 0.60 \\
\hline $\mathrm{CRO}$ & 0.11 & 0.73 & 0.22 & 1.00 & 0.22 & 0.00 & 0.11 & 0.58 & 0.33 & 0.70 & 0.60 \\
\hline AUS & 0.11 & 0.91 & 0.22 & 1.00 & 0.22 & 0.04 & 0.11 & 0.31 & 0.33 & 0.70 & 0.60 \\
\hline SLV & 0.11 & 0.73 & 0.22 & 1.00 & 0.22 & 0.01 & 0.11 & 0.38 & 0.33 & 0.70 & 0.58 \\
\hline $\mathrm{CZR}$ & 0.11 & 0.73 & 0.22 & 1.00 & 0.22 & 0.00 & 0.11 & 0.35 & 0.33 & 0.70 & 0.57 \\
\hline SWE & 0.11 & 0.82 & 0.22 & 0.99 & 0.22 & 0.00 & 0.11 & 0.25 & 0.33 & 0.70 & 0.57 \\
\hline $\mathrm{BOL}$ & 0.11 & 0.73 & 0.22 & 1.00 & 0.22 & 0.09 & 0.11 & 0.82 & 0.33 & 0.30 & 0.51 \\
\hline LUX & 0.11 & 0.73 & 0.22 & 1.00 & 0.22 & 0.23 & 0.11 & 0.54 & 0.33 & 0.30 & 0.51 \\
\hline IRL & 0.11 & 0.82 & 0.22 & 1.00 & 0.22 & 0.19 & 0.11 & 0.53 & 0.33 & 0.30 & 0.51 \\
\hline LIT & 0.11 & 0.73 & 0.22 & 0.97 & 0.22 & 0.00 & 0.11 & 0.64 & 0.33 & 0.30 & 0.47 \\
\hline SLO & 0.11 & 0.82 & 0.22 & 0.98 & 0.22 & 0.06 & 0.11 & 0.41 & 0.33 & 0.30 & 0.47 \\
\hline MLT & 0.11 & 0.27 & 0.22 & 0.98 & 0.22 & 0.00 & 0.11 & 0.93 & 0.33 & 0.30 & 0.45 \\
\hline EST & 0.11 & 0.55 & 0.22 & 0.96 & 0.22 & 0.01 & 0.11 & 0.45 & 0.33 & 0.30 & 0.42 \\
\hline CYP & 0.11 & 0.46 & 0.22 & 1.00 & 0.22 & 0.00 & 0.11 & 0.39 & 0.33 & 0.30 & 0.42 \\
\hline LAT & 0.11 & 0.55 & 0.22 & 0.95 & 0.22 & 0,00 & 0.11 & 0.34 & 0.33 & 0.30 & 0.41 \\
\hline \begin{tabular}{|l|} 
EU-28 \\
\end{tabular} & 0.11 & \begin{tabular}{l|l}
0.74 \\
\end{tabular} & 0.22 & 0.99 & 0.22 & 0.05 & 0.11 & 0.51 & 0.33 & \begin{tabular}{l|}
0.65 \\
\end{tabular} & 0.59 \\
\hline
\end{tabular}

Source: Eurostat (2013-2015); authorial calculations 
Ranking of the analyzed countries by groups (FDG1, FDG2 or FDG3) according to CIFD value is presented in Figure 3.

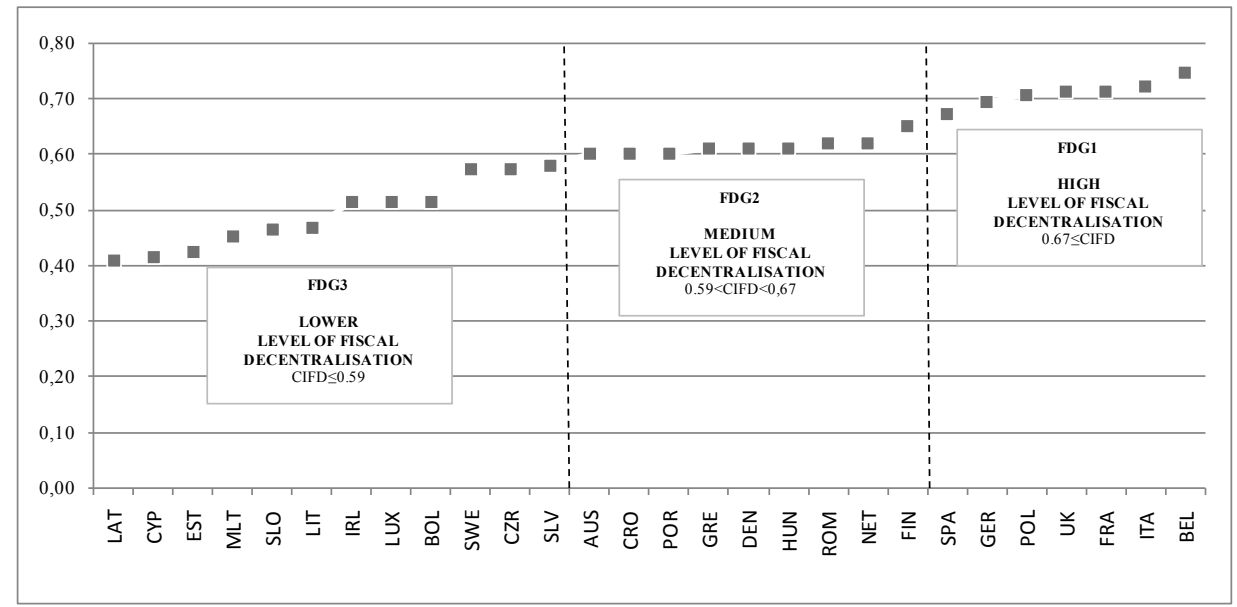

Figure 3: Fiscal decentralization level for EU-28 countries by CIFD value

Source: The authors

In the present paper's framework, we assumed that CIFD represents a model of an actual state and consists of parameters (indicators) with possible variable values in different observed time periods. It is worth adding another assumption: the existing fiscal decentralization system in each particular country achieves the highest possible level of real effectiveness if it reflects a high level of fiscal decentralization and a high level of compliance with the ECLSG guidelines. Using CIFD, we conducted a direct international comparison of the various EU-28 fiscal decentralization systems. For the studied countries, we have obtained the result in the form of identifiable levels of fiscal decentralization, which serve as a basis for assessing the achievement of the actual effectiveness of the already existing fiscal decentralization systems. Given that there is a direct link between the CIFD and the quantified core principles of ECLSG, the CIFD indicators themselves point to either greater or lesser compliance of their fiscal decentralization systems with the selected ECLSG' core principles.

On the basis of the calculated values of each of the CIFD indicators, we placed the EU-28 member states in one of the groups according to the achieved level of compliance with the studied basic principle of ECLSG or the degree of decentralization according to the number of local government units (hereinafter: LGU). The first group (group 1, hereinafter: S1) therefore consists of countries which, according to the indicator value, achieve a high level of compliance with the studied basic principle of ECLSG or a high degree of decentralization according to the number of LGUs; the second group (group 2, hereinafter: S2) consists of countries with a medium degree of coherence with the studied basic principle of ECLSG or an average degree of decentralization according to the number of LGUs; the third group (group 3, hereinafter S3) comprises countries with a low degree of compliance with the studied basic prin- 
ciple of ECLSG or a low degree of decentralization according to the LGU number. At individual CIFD indicators, we have set limit values for placement of the studied country to S1 $\left(\mathrm{IF}_{\mathrm{i}}=0.909, \mathrm{IS}_{\mathrm{i}}=1.000, \mathrm{IA}_{\mathrm{i}}>0.082, \mathrm{II}_{\mathrm{i}}>0.419, \mathrm{IR}_{\mathrm{i}}=1.000\right), \mathrm{S} 2\left(0.744 \leq \mathrm{IF}_{\mathrm{i}}<0.909\right.$, $\left.0.990 \leq \mathrm{IS}_{\mathrm{i}}<1.000,0<\mathrm{IA}_{\mathrm{i}} \leq 0.082,0.340<\mathrm{II}_{\mathrm{i}} \leq 0.419, \mathrm{IR}_{\mathrm{i}}=0.700\right)$ and $\mathrm{S} 3\left(\mathrm{IF}_{\mathrm{i}}<0.744, \mathrm{IS}_{\mathrm{i}}<0.990\right.$, $\mathrm{IA}_{\mathrm{i}}=0, \mathrm{II}_{\mathrm{i}} \leq 0.340, \mathrm{IR}_{\mathrm{i}}=0.300$ ).

We presume that the value of the country's placement to S1 is 3 points for each of the indicators, to S2 2 points and to S3 1 point. This means that the higher the value of each country relative to the estimated number of points, the higher the degree of compliance with the examined basic principles of ECLSG or that the degree of decentralization according to the number of LGUs is reached by the examined system of fiscal decentralization in this country.

A threshold value among countries with a high level of compliance with the studied basic principles of ECLSG or a high degree of decentralization according to the number of LGUs (high compliance of existing fiscal decentralization systems with the ECLSG 1 guidelines, hereinafter SSM1) and countries with a medium level of compliance with the studied basic principles of ECLSG or an average degree of decentralization in terms of LGU number (medium compliance of existing fiscal decentralization systems with the ECLSG 2 guidelines, hereinafter referred to as SSM2) is represented by countries ranked in most indicators (four out of five) to S1. Countries where the value of total placement points in one of the groups $(\mathrm{S} 1, \mathrm{~S} 2, \mathrm{~S} 3)$ is equal to or less than the calculated average of all EU-28 points (9.8) are classified in a group of countries with a low degree of compliance with the studied basic principles of ECLSG or a low degree of decentralization according to the number of LGUs (low compliance of existing fiscal decentralization systems with the ECLSG 3 guidelines, hereinafter: SSM3). The EU-28 member states placement in one of the three groups (SSM1, SSM2, and SSM3) is shown in Table 2.

As seen in Table 2, only two of the EU-28 are ranked to S1 in four out of five CIFD indicators; Italy and Belgium consequently achieve high compliance of their existing fiscal decentralization systems with the ECLSG (SSM1) guidelines. On the other hand, 11 of the EU-28 member states are ranked to SSM3 in terms of the number of points of the placement to S1, S2 and S3. In spite of signing of ECLSG and the commitment to comply with the Charter, the fundamental principles or guidelines for the regulation and operation of their LGU's are not respected. Given the low compliance of existing fiscal decentralization systems with the ECLSG guidelines, these countries are far from the establishment of an effective existing fiscal decentralization system.

As seen in Table 2, only two of the EU-28 are ranked to S1 in four out of five CIFD indicators; Italy and Belgium consequently achieve high compliance of their existing fiscal decentralization systems with the ECLSG (SSM1) guidelines. On the other hand, 11 of the EU-28 member states are ranked to SSM3 in terms of the number of points of the placement to S1, S2 and S3. In spite of signing of ECLSG and the commitment to comply with the Charter, the fundamental principles or guidelines for the regulation and operation of their LGU's are not respected. Given the low compliance 
Table 2: EU-28 placement in one of the three groups (SSM1, SSM2, SSM3)

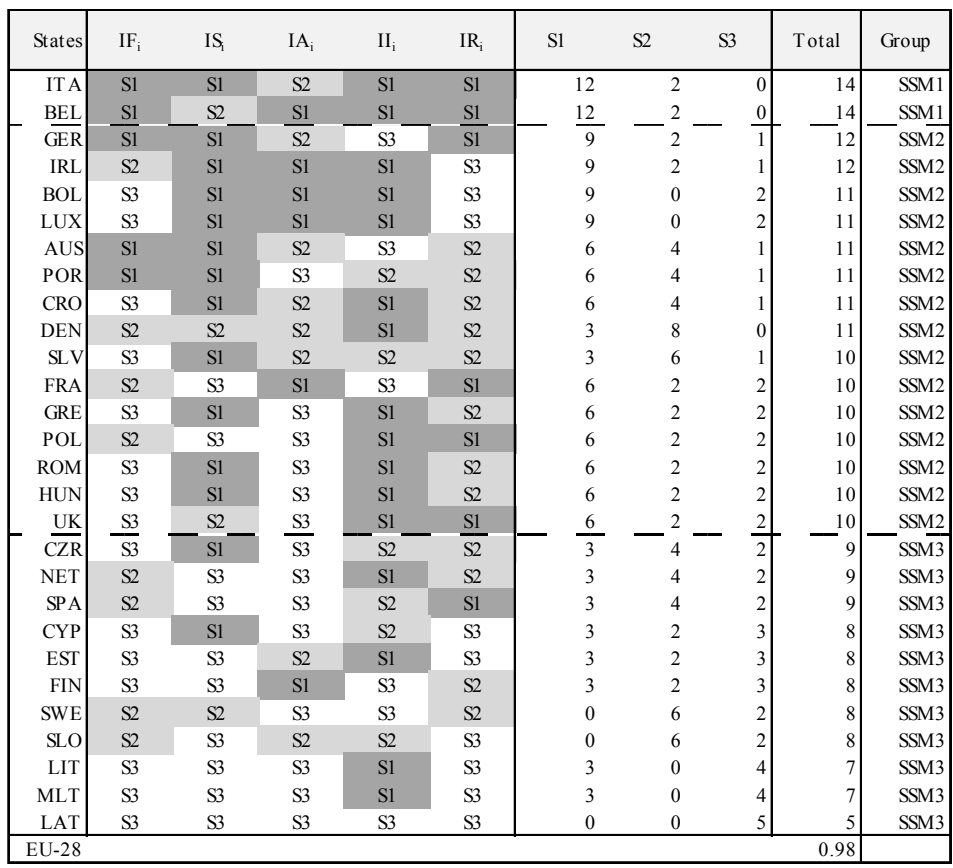

Source: Authorial computations

of existing fiscal decentralization systems with the ECLSG guidelines, these countries are far from the establishment of an effective existing fiscal decentralization system.

26 out of a total of 28 countries are classified as SSM2 ( 15 countries) and SSM3 (11 countries). This implies that the fiscal decentralization systems of these countries are not fully in line with the ECLSG guidelines. In the case of Italy and Belgium, despite being categorized as SSM1, we cannot confirm that their existing fiscal decentralization systems are fully in line with the studied fundamental principles of ECLSG as both countries are ranked to $\mathrm{S} 2$ in one of the CIFD indicators, namely Italy at $\mathrm{IA}_{\mathrm{i}}$ and Belgium at IS. Based on these findings, an individual country can detect the weakness of its own fiscal decentralization system and take certain steps to upgrade it in the direction of greater efficiency.

As mentioned earlier, one of the most important assumptions of our contribution is the assumption of achieving real effectiveness. In continuation, we will thus check whether each country, which is classified as SFD1 according to the CIFD value, is also ranked as SSM1 according to the achieved level of compliance with the individual fundamental principles of ECLSG or the degree of decentralization according to the number of LGUs. The latter is shown in Figure 4. 


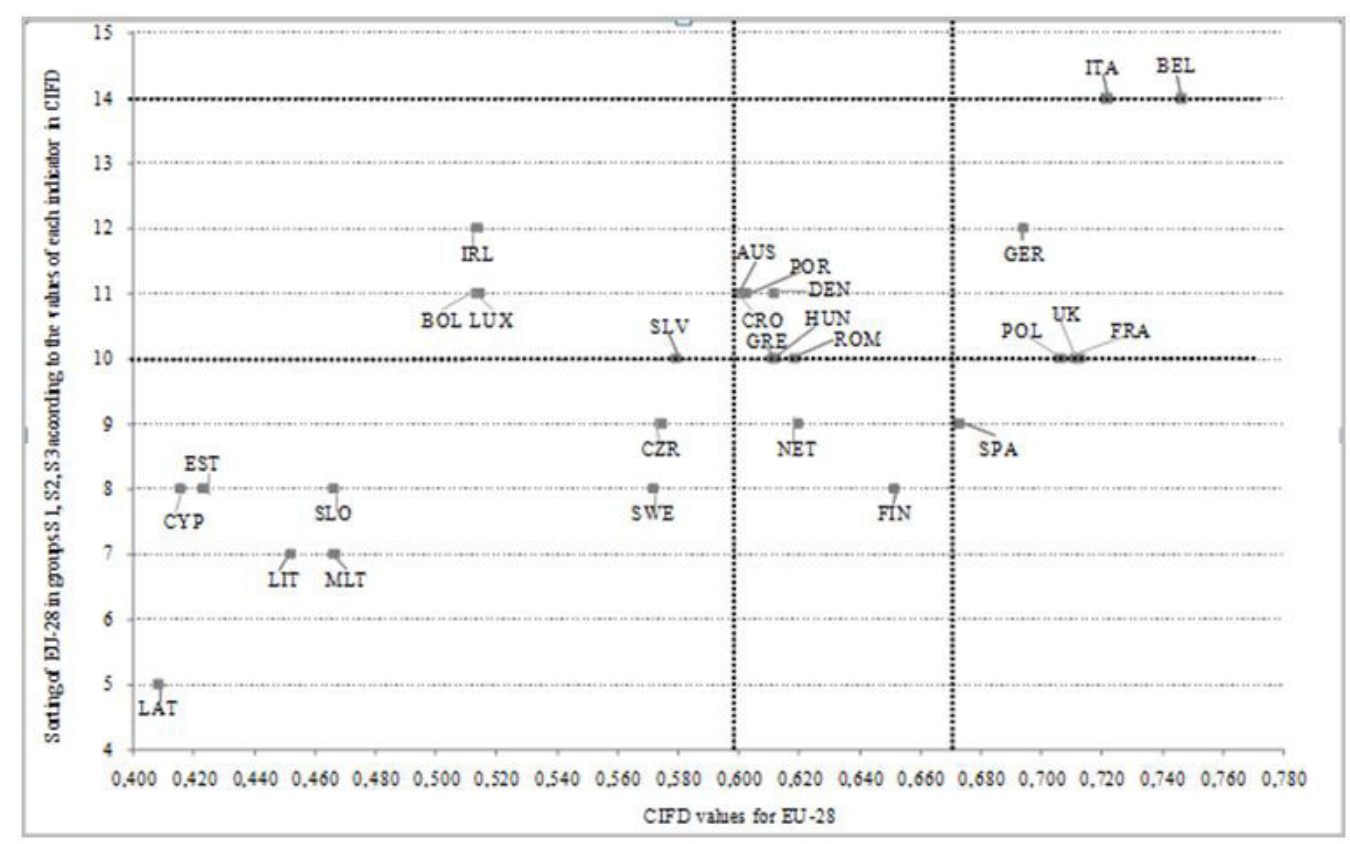

Figure 4: Achieving substantive effectiveness of the existing EU-28 fiscal decentralization systems

Source: The authors

As seen in Figure 4, EU-28 countries are, on the horizontal axis, classified into one of the groups (SFD1, SFD2, SFD3) according to the CIFD limit values. A country is listed to SFD1 if the CIFD value is equal to or greater than 0.67, to SFD2 if the CIFD is lower than 0.67 and greater than or equal to 0.59 , and to SFD3 if the CIFD value is lower than 0.59. On the vertical axis the EU-28 countries are classified into one of the groups (SSM1, SSM2, SSM3) according to the evaluation of the achieved level of compliance with the individual fundamental principles of ECLSG or the degree of decentralization according to the number of LGUs.

A brief EU-28 review shows that Italy and Belgium demonstrate a high level of real effectiveness of existing fiscal decentralization systems, reflecting a high degree of fiscal decentralization (SFD1) and high compliance with the ECLSG (SSM1) guidelines. On the other hand, countries such as Cyprus, Czech Republic, Estonia, Latvia, Lithuania, Malta, Slovenia and Sweden demonstrate a low level of actual efficiency of existing fiscal decentralization systems, since they are classified to SFD3 according to the CIFD value, and also achieve low level compliance with the ECLSG guidelines (SSM3). Countries that demonstrate medium level of substantive effectiveness are Austria, Denmark, Greece, Croatia, Hungary, Portugal and Romania. These countries are classified as SFD2 according to the CIFD value and to SSM2 according to the mean level of compliance with the ECLSG guidelines. On this basis, for a total of 17 countries of all EU-28 countries, we can confirm that, according to the level of fiscal decentralization (CIFD) and the achieved level of compliance with the fundamental principles of ECLSG or the degree of decentralization according to the number 
of LGUs, they are classified to the same group according to the level of compliance within the observed time period, i.e. to SFD1 and SSM1 (2 countries), SFD 2 and SSM2 (7 countries), and SFD3 and SSM3 (8 countries).

Furthermore, we notice that countries such as France, Germany, Poland and UK achieve a high level of fiscal decentralization with respect to the CIFD (SFD1) value while achieving a medium level of compliance with the ECLSG (SSM2) guidelines. For these countries, we can affirm that the potential of their existing fiscal decentralization systems is high in order to achieve a high level of actual efficiency if they eliminate certain recognized deficiencies of such systems. The disadvantages of already existing fiscal decentralization systems can be eliminated if the country, in the case of certain indicators constituting the CIFD, demonstrates a medium or high (instead of low) degree of compliance with the studied fundamental principles of ECLSG. For example, UK is ranked to $\mathrm{S} 3$ at $\mathrm{IF}_{\mathrm{i}^{\prime}}$ France and Poland at $\mathrm{IS}_{\mathrm{i}^{\prime}}$ Poland and $\mathrm{UK}$ at $\mathrm{IA}_{\mathrm{i}}$ and Germany and France at II.

The Netherlands and Finland are countries that are classified to SFD2 according to the CIFD value and to SSM3 according to the achieved degree of compliance with the ECLSG guidelines. These two countries are therefore far away from achieving a high level of substantive effectiveness. Both countries demonstrate a low level of compliance with 'the principle of coherence of financial resources of LGU with their competencies' $\left(\mathrm{IS}_{\mathrm{i}}\right)$. In addition, Netherlands is also ranked to $\mathrm{S} 3$ according to the $\mathrm{IA}_{\mathrm{i}}$ value, while Finland shows a low level of compliance with 'the principle of flexibility' $\left(\mathrm{IF}_{\mathrm{i}}\right)$ and 'the balancing principle' $\left(\mathrm{II}_{\mathrm{i}}\right)$.

Countries, such as Bulgaria, Ireland, Luxembourg and Slovakia, are classified as SFD3 according to the CIFD value and to SSM2 according to the achieved level of compliance with the studied fundamental principles of ECLSG. Consequently, these countries are far from achieving a high level of substantive effectiveness of their already existing fiscal decentralization systems.

In the fourth part of the paper, we will present a simulation of the overall empirical CMFD, based on the findings of a direct international comparison of different EU-28 fiscal decentralization systems. Such a model can serve as a tool for countries to upgrade their already existing system of fiscal decentralization in order to achieve a high level of substantive effectiveness.

\section{Simulation of the empirical conceptual model of fiscal decentralization (CMFD)}

The purpose of this section is to present a simulation of the empirical CMFD that reflects a high degree of fiscal decentralization and a high level of compliance with the fundamental principles of ECLSG $\left(\mathrm{IF}_{\mathrm{i}^{\prime}} \mathrm{IS}_{\mathrm{i}^{\prime}} \mathrm{IA}_{\mathrm{i}^{\prime}} \mathrm{II}_{\mathrm{i}}\right.$ ) or a high degree of decentralization in terms of LGU number $\left(\mathrm{IR}_{\mathrm{i}}\right)$. We will constantly strive to build a robust model of fiscal decentralization, which is innovative and stable at the same time. This means that such a model is also useful for changing parameter (indicator) values in the selected time periods. In addition, the empirical CMFD built on this basis should serve 
as an instrument for the individual countries to upgrade their already existing system of fiscal decentralization in order to achieve greater efficiency.

The simulation of the empirical CMFD, as an instrument for upgrading the already existing system of fiscal decentralization in each country in the direction of reaching a high level of efficiency, is first presented as an image (Figure 5), followed by a detailed description.

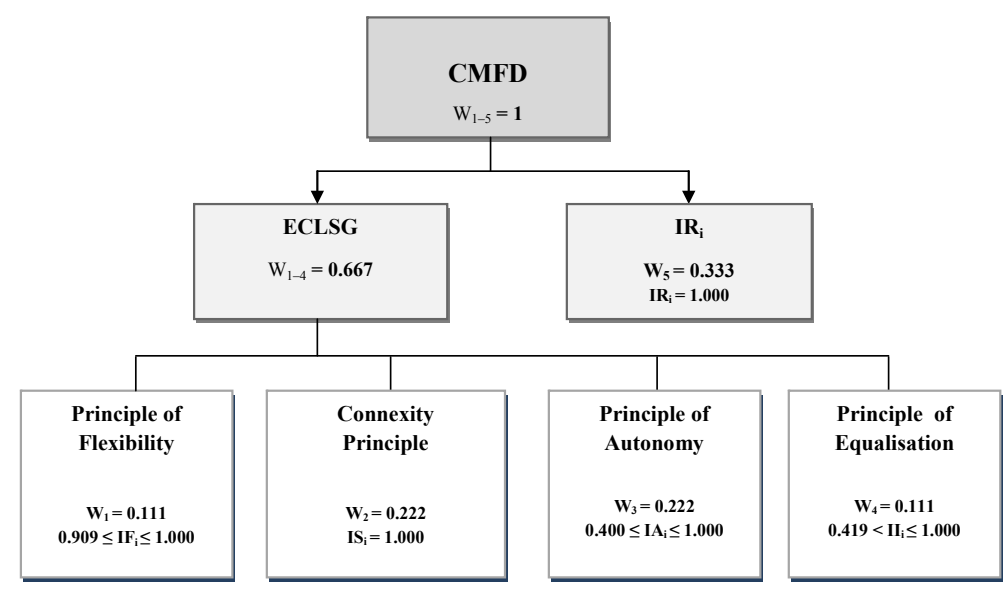

Figure 5: Simulation of the empirical CMFD

Source: The authors

The empirical CMFD formula for the $\mathrm{i}$-th country is written as follows:

$\mathrm{CMFD}_{\mathrm{i}}=0.111 \times \mathrm{IF}_{\mathrm{i}}+0.222 \times \mathrm{IS}_{\mathrm{i}}+0.222 \times \mathrm{IA}_{\mathrm{i}}+0.111 \times \mathrm{II}_{\mathrm{i}}+0.333 \times \mathrm{IR}_{\mathrm{i}} \quad$ (2), where:

$\mathrm{CMFD}_{\mathrm{i}}$ - simulation of the empirical conceptual model of fiscal decentralization of the i-th country;

$\mathrm{W}_{1-5}$ - weighting the significance of individual CMFD indicators, where the total value $\mathrm{W}=1$;

$\mathrm{IF}_{\mathrm{i}} \quad$ - indicator of flexibility of the i-th country with a value of 0.909-1;

IS $_{i} \quad$ - indicator of compliance of financial resources of LGU with the powers of the LGU of the i-th country with a value of 1 ;

$\mathrm{IA}_{i} \quad$ - indicator of the autonomy of the i-th country with a value of 0.400-1;

$\mathrm{II}_{\mathrm{i}} \quad-$ a level indicator of the $\mathrm{i}$-th country with a value greater than 0.419 up to 1 ;

$I_{i} \quad$ - indicator of LGU number of the i-th country with a value of 1 .

The empirical CMFD consists of a total of five indicators $\left(\mathrm{IF}_{\mathrm{i}^{\prime}} \mathrm{IS}_{\mathrm{i}^{\prime}} \mathrm{IA}_{\mathrm{i}^{\prime}} \mathrm{II}_{\mathrm{i}^{\prime}} \mathrm{IR}_{\mathrm{i}}\right)$, the indicators and weightings of each of them in the composition of CMFD are identical to those of the CIFD. The main difference between CMFD and CIFD is thus in the definition of the limit values of single indicators $\left(\mathrm{IF}_{\mathrm{i}^{\prime}} \mathrm{IS}_{\mathrm{i}^{\prime}} \mathrm{IA}_{\mathrm{i}^{\prime}} \mathrm{II}_{\mathrm{i}^{\prime}} \mathrm{IR}_{\mathrm{i}}\right)$, which indicate the achievement of a high degree of efficiency of the fiscal decentralization system of the i-th country. 
Based on all the findings presented in the paper, we conclude that the upgrading of the already existing system of fiscal decentralization of the i-th country by achieving a high level of efficiency is possible, if such a system, in a designated time period, is formed as follows:

- $0.909 \leq \mathrm{IF}_{\mathrm{i}} \leq 1.000$. LGU of the i-th country show revenues from at least 10 of the total of all 11 identified revenue groups $(0.909 \leq \mathrm{IFi} \leq 1,000)$. The financial system of this country is diverse and flexible enough to follow the actual movement of the needs of local communities and is, consequently, effective;

- $\mathrm{IS}_{\mathrm{i}}=1.000$. LGU of the $\mathrm{i}$-th country shows more revenue than expenditure $(\mathrm{ISi}=1.000)$. The financial resources of the LGU of the i-th country are thus in line with their respective competences and the tasks assigned to them by the law. Consequently, such a system of fiscal decentralization of the i-th state is effective; $-0.400 \leq \mathrm{IA}_{\mathrm{i}} \leq 1.000$. The presented share of autonomous tax revenues of the LGU of the i-th country in their total revenue amounts to 0.400 or higher $(0.400 \leq \mathrm{IAi} \leq$ 1.000). LGUs of the i-th country are thus entitled to an adequate part of their own financial resources in order to be able to autonomously propose taxes and taxation and, in the exercise of their powers, freely dispose of them without any restriction by the CRU. This reflects an effective system of fiscal decentralization of the i-th country;

$-0.419<\mathrm{II}_{\mathrm{i}} \leq 1.000$. The presented share of transfers of LGUs of the $\mathrm{i}$-th country in their total revenue amounts to over $0.419(0.419<\mathrm{Ili} \leq 1.000)$. The choice of a threshold at this level is consistent with the choice of the threshold of achieving a high level of compliance for each country in the context of verification of the balancing principle. The fiscal decentralization system of the i-th country is effective if, for the protection of its LGUs, which are financially weaker, appropriate compensatory mechanisms and measures that eliminate the consequences of unequal distribution of available financial resources between individual LGUs (in this case, transfers) are used;

- $\mathrm{IR}_{\mathrm{i}}=1.000$. The existing system of fiscal decentralization has a three-level LGU structure (usually municipalities, districts, regions, etc.) $\left(\mathrm{IR}_{\mathrm{i}}=1.000\right)$. Paragraph 3 of Article 4 of ECLSG states, inter alia, that public tasks should be exercised by those authorities which are closest to citizens. We have hypothesized, on this basis, that the greater the number of the i-th country's LGU, the closer can we get to the actual needs of the environment or to the local needs of citizens, which, at the same time, reflects the effective system of fiscal decentralization of the i-th country.

The obtained value of the empirical CMFD of the i-th country with the inserted limit values of individual indicators to achieve a high degree of efficiency of its fiscal decentralization system is as follows:

$\mathrm{CMFD}_{\mathrm{i}}=0.111 \times 0.909+0.222 \times 1+0.222 \times 0.400+0.111 \times 0.419+0.333 \times 1$ (3), with $\mathrm{CMFD}_{\mathrm{i}}=0.791$. 
For a country with CMFD value equal to or greater than 0.791 , we can confirm that its existing fiscal decentralization system achieves a high level of efficiency as it reflects respect for the most important ECLSG guidelines. On the other hand, the more the country is distant from the CMFD value and, consequently, from the limit values for achieving a high degree of efficiency for individual indicators, the greater changes must be used in the very functioning of the existing fiscal decentralization system.

In the literature published to date this kind of simulation of the empirical CMFD as a tool for upgrading the existing system of fiscal decentralization in individual countries towards reaching a higher level of efficiency was not detected. We can therefore conclude that the presented model is innovative. In addition to innovation, the empirical CMFD has some other advantages, such as robustness and stability. This means that such a model can also be used in the event of changes in the value of individual indicators in selected different observation periods of the studied country. Each country can thus determine the critical points or deficiencies of the existing system of fiscal decentralization in the light of the comparison of the value of a single indicator with the raised limit value for achieving a high level of efficiency within the empirical CMFD; the awareness/knowledge of these is the basis for the adoption of certain measures that the country should use in order to achieve highly efficient and sustained fiscal decentralization in the long run.

\section{Conclusion}

In the first part of the paper we presented the main emphasis of the authors of first and second generation. Furthermore, we were interested in what methods or indicators the authors used in the literature for achieving an international comparison of different systems of fiscal decentralization. In order to calculate the value of individual indicators, the authors have often used classical statistical databases which cannot answer some of the key issues of fiscal decentralization (the issue of autonomy of revenue and income, vertical fiscal balance, etc.). Research results obtained on this basis are not sufficient to provide an unequivocal overview of the actual state of fiscal decentralization in each country, which greatly complicates their direct comparability. The implementation of a direct international comparison of differently designed fiscal decentralization systems of the studied countries with the appropriate common denominator was thus the fundamental goal of our study.

In the search for a common denominator for the implementation of a direct international comparison of the existing fiscal decentralization systems of the countries included in the sample (EU-28), we continually strived for its composition to be an improvement of the classical methods (indicators) that are used to measure fiscal decentralization, and, at the same time, to reflect consideration of the selected fundamental principles of ECLSG. Bearing this in mind, we have created CIFD, which consists of five indicators, four of which represent quantified selected fundamental principles of ECLSG (the principle of flexibility, the principle of coherence of financial resources of LGU with their competencies, the principle of autonomy and the prin- 
ciple of equalization). The fifth indicator of CIFD, the number of LGUs, also follows from the ECLSG guidelines, namely the third paragraph of Article 4.

One of the most important hypotheses of this study is that of achieving substantive effectiveness, namely that 'the existing fiscal decentralization system in each country achieves higher level of substantive effectiveness, if it reflects a high level of fiscal decentralization and high compliance with the ECLSG guidelines'. For the EU-28, we thus checked that each country, which is classified as SFD1 according to the CIFD value, is also ranked as SSM1 according to the achieved level of compliance with the ECLSG guidelines. In this respect, for 17 countries of all of the EU-28 it was confirmed that, according to the level of fiscal decentralization (CIFD) and the achieved level of compliance with the fundamental principles of ECLSG or the degree of decentralization according to the number of LGUs, they are classified in the same group according to the demonstrated degree of coherence within the observation period, namely to SFD1 and SSM1 (2 countries), to SFD 2 and SSM2 (7 countries), and to SFD3 and SSM3 (8 countries).

We furthermore discovered that countries such as France, Germany, Poland and UK achieve a high level of fiscal decentralization with respect to the CIFD (SFD1), while achieving a medium level of compliance with the ECLSG (SSM2) guidelines. For these countries, the potential of their existing fiscal decentralization systems to achieve a high level of actual efficiency is high, if they eliminate certain recognized deficiencies of such systems. Nederland and Finland are countries that are classified as SFD2 according to the CIFD value and to SSM3 according to the achieved level of compliance with the ECLSG guidelines, which implies a significant detachment of their existing fiscal decentralization systems from achieving a high level of substantive effectiveness. Similarly, this applies to countries such as Bulgaria, Ireland, Luxembourg and Slovakia, which are classified as SFD3 according to the CIFD value and to SSM2 according to the achieved level of compliance with the studied ECLSG guidelines. When observing the level of substantive effectiveness of all EU-28, Spain stands out, since it is classified as SFD1 according to the CIFD value, while ranked to SSM3 according to the value of the compliance points with individual CIFD indicators. Despite the fact that Spain is a country implementing a three-level structure of LGU and consequently classified as $S 1$ in $\mathbb{I R}_{\mathrm{i}^{\prime}}$ the principle of coherence of financial resources of LGU with their competencies $\left(\mathrm{IS}_{\mathrm{i}}\right)$ and the principle of autonomy of the LGU ( $\left(A_{i}\right)$ show a low level of compliance (S3).

In the fourth part of the paper we presented a simulation of the empirical CMFD. The empirical CMFD simulation consists of five indicators $\left(\mathrm{IF}_{\mathrm{i}^{\prime}} \mathrm{IS}_{\mathrm{i}^{\prime}} \mathrm{IA}_{\mathrm{i}^{\prime}} \mathrm{II}_{\mathrm{i}^{\prime}} \mathrm{IR}_{\mathrm{i}}\right)$ with the indicators and weights of importance of each of them identical to those of the CIFD. For the empirical CMFD, the limit value for achieving a high degree of efficiency of the fiscal decentralization system of the i-th country was determined for each of the five indicators. This is, at the same time, the main difference between the empirical CMFD and CIFD. The existing system of fiscal decentralization of the i-th country achieves a high level of efficiency if the value, obtained with empirical CMFD, 
is equal to or greater than 0.791 . In our opinion, such fiscal decentralization system reflects respect for the most important ECLSG guidelines. In contrast, the further the i-th country is from the threshold of achieving a high degree of efficiency of the existing fiscal decentralization system, obtained from the CMFD (0.791), the lesser the compliance and respect for the ECLSG guidelines.

Each country has now the opportunity to identify critical points or deficiencies of its own fiscal decentralization system by comparing the value of a particular indicator with a raised limit value for this to achieve a high degree of efficiency within the empirical CMFD simulation. Recognizing the shortcomings of the already existing fiscal decentralization system presents an opportunity for each country and, at the same time, a basis for the adoption of certain measures that will lead to the achievement of a transparent, sustainable and highly efficient fiscal decentralization system in the long run. For this reason, we believe in great importance of further in-depth research in the area of fiscal decentralization.

\section{References:}

1. Besley, T. and Coate, S., 'Centralized versus Decentralized Provision of Public Goods: A Political Economy Approach', 2003, Journal of Public Economics, vol. 87, no. 12, pp. 2611-2637.

2. Boex, J., 'Fiscal Decentralization and Intergovernmental Finance Reform as an International DevelopmentStrategy', 2009, IDG Working Paperno. 2009-06, [Online] available at http://citeseerx.ist.psu.edu/viewdoc/download?doi=10.1.1.515.2671\&rep=rep1\&type= pdf, accessed on March 18, 2018.

3. Bole, V. and Jere, Ž., 'Prihodki nižjih ravni države (Revenues at Lower Governmental Levels)', 2005, Lex localis - Journal of Local Self-Government, vol. 3, no. 4, pp. 93-117.

4. Brezovnik, B. and Oplotnik, Z.J., Fiskalna decentralizacija v Sloveniji - oris stanja, mednarodna primerjava in problematika (Fiscal Decentralization in Slovenia - State of the Art, International Comparison and Problems), Maribor: Inštitut za lokalno samoupravo in javna naročila Maribor, 2003.

5. Buchanan, J.M., 'An Economic Theory of Clubs', 1965, Economica, vol. 32, no. 125, pp. $1-14$.

6. Cheikbossian, G., 'Rent-seeking, Spillovers and the Benefits of Decentralization', 2008, Journal of Urban Economics, vol. 63, no. 1, pp. 217-228.

7. ECLSG, European Charter of Local Self-Government, 1996, Official Gazette of the Republic of Slovenia, no. 57/1996.

8. European Commission, 'Annex B - Methodology and Explanatory Notes', 2012, [Online] available at http://ec.europa.eu/taxation_customs/resources/documents/taxation/ gen_info/economic_analysis/tax_structures/2012/methodology.pdf, accessed on November 4, 2014.

9. European Commission, 'Taxes in Europe Database', 2016, [Online] available at http:// ec.europa.eu/taxation_customs/tedb/taxSearch.html, accessed on May 2, 2016.

10. Eurostat, 'European System of Accounts - ESA 2010', 2013, Luxembourg: Publications Office of the European Union, [Online] available at https://ec.europa.eu/eu 
rostat/documents/3859598/5925693/KS-02-13-269-EN.PDF/44cd9d01-bc64-40e5-bd40d17df0c69334, accessed on March 20, 2018.

11. Eurostat, 'Government Revenue, Expenditure and Main Aggregates', 2014, [Online] available at http://appsso.EUROSTAT.ec.europa.eu/nui/show.do?dataset=gov_a_mai n\&lang=en, accessed on September 1, 2014.

12. Eurostat, 'Population in EU-28', 2015, [Online] available at http://appsso.EUROSTAT. ec.europa.eu/nui/show.do, accessed on February 1, 2016.

13. Finžgar, M. and Oplotnik, Ž.J., 'Comparison of Fiscal Decentralization Systems in EU27 According to Selected Criteria', 2013, Lex localis - Journal of Local Self-Government, vol. 11, no. 3, pp. 651-672.

14. Musgrave, R.A., The Theory of Public Finance: A Study in Public Economy, New York: McGraw-Hill, 1959.

15. Oates, W.E., Fiscal Federalism, New York: Harcourt Brace Jovanovich, 1972.

16. Olson, M., 'The Principle of Fiscal Equivalence: The Division of Responsibilities among Different Levels of Government', 1969, The American Economic Review, vol. 59, no. 2, pp. 479-487.

17. Samuelson, P., 'The Pure Theory of Public Expenditure', 1954, Review of Economics and Statistics, vol. 36, no. 4, pp. 387-389.

18. Sandler, T. and Tschirhart, J., 'Club Theory: Thirty Years Later', 1997, Public Choice, vol. 93, no. 3/4, pp. 335-355.

19. Seabright, P., 'Accountability and Decentralisation in Government: An Incomplete Contract Model', 1996, European Economic Review, vol. 40, no. 1, pp. 61-89.

20. Slavinskaite, N., 'Fiscal Decentralization and Economic Theory', 2015, [Online] available at https://recipp.ipp.pt/bitstream/10400.22/7968/3/04_A_Neringa\%20Slavinskaite. pdf, accessed on January 29, 2017.

21. Tiebout, C.M., 'A Pure Theory of Local Expenditures', 1956, Journal of Political Econo$m y$, vol. 64, no. 5, pp. 416-424.

22. Vo, D.H., 'The Economics of Fiscal Decentralization', 2010, Journal of Economics Surveys, vol. 24, no. 4, pp. 657-679.

23. Wagner, R.E., Fiscal Sociology and the Theory of Public Finance: An Exploratory Essay, Cheltenham: Edward Elgar, 2007.

24. Weingast, B.R., 'The Economic Role of Political Institutions: Market-preserving Federalism and Economic Development', 1995, Journal of Law, Economics and Organization, vol. 11, no. 1, pp. 1-31. 